\title{
Microtearing Turbulence Limiting the JET-ILW Pedestal
}

\author{
D.R. Hatch, ${ }^{1}$ M. Kotschenreuther, ${ }^{1}$ S. Mahajan, ${ }^{1}$ P. Valanju, ${ }^{1}$ F. Jenko, ${ }^{2}$ D. Told,${ }^{2}$ T. Görler,${ }^{3}$ and S. Saarelma ${ }^{4}$ \\ ${ }^{1}$ Institute for Fusion Studies, University of Texas at Austin, Austin, Texas, 78712 \\ ${ }^{2}$ Department of Physics and Astronomy, University of California, Los Angeles, CA 90095, USA \\ ${ }^{3}$ Max Planck Institute for Plasma Physics, Boltzmannstr. 2, 85748 Garching, Germany \\ ${ }^{4}$ Culham Centre for Fusion Energy, Culham Science Centre, Abingdon OX14 3DB, United Kingdom
}

\begin{abstract}
Gyrokinetic simulations identify microtearing modes (MTM) to be the dominant microinstabilities in the JET-ILW (ITER like wall) pedestal. Nonlinear simulations show that MTM-driven turbulence produces the bulk of the transport in the steep gradient region, demonstrating that MTM may be the principal mechanism limiting JET-ILW pedestal evolution. The combination of MTM, electron temperature gradient (ETG), and neoclassical transport reproduces experimental power balance across most of the pedestal. Kinetic ballooning modes are significant only in the local limit and only at low $\beta$, far below the experimental operating point.
\end{abstract}

PACS numbers:

The tokamak H-mode [1] is defined by a narrow insulating region - the pedestal - at the plasma edge, where turbulence is suppressed and sharp pressure gradients develop. The pedestal is at the center of the most pressing issues facing fusion energy. ITER, for example, must reach a sufficient temperature at the pedestal's inner boundary in order to achieve its fusion power targets [2]. This work reports the results of, perhaps, the very first, first-principles simulations of the H-mode pedestal dynamics that reproduce experimentally observed transport levels. In addition to providing unprecedented insight into the dynamics of the existing $\mathrm{H}$-mode pedestals, such simulations are likely to advance our capabilities towards predictive modeling of future burning plasma devices.

This study targets the JET-ILW (ITER-like wall) pedestal [4-6], which approaches ITER conditions in two important ways: 1) as the largest tokamak in operation, it most-closely approximates plasma parameters that are dependent on machine size (like $\rho_{*}$, the ratio of the gyroradius to minor radius), 2) to approximate ITER conditions even better, JET has recently installed an ITERlike wall (ILW) (composed of a tungsten divertor and beryllium chamber). This modification decreases the global performance of discharges by $20-30 \%$, attributable largely to changes in pedestal structure. In addition to the performance loss, certain observed key properties of the ILW pedestal are inconsistent with predictions of the leading pedestal model (EPED [7, 8]).

In this work, we elucidate possible mechanisms limiting profile evolution in the JET-ILW discharges. We demonstrate, through simulations using the gyrokinetic code GENE $[9,10]$, that the microtearing mode (MTM) [11-14] is the dominant instability in the pedestal. Interestingly, the simulations do not find the kinetic ballooning mode (KBM), a basic component of the EPED model, except locally in a narrow region near the separatrix. Most importantly, we determine via nonlinear gyrokinetic simulations that a combination of MTM and electron temperature gradient (ETG) $[9,15-18]$ driven turbulence plus the neoclassical flux, produces transport levels closely matching experimental power balance across most of the pedestal, demonstrating the capacity of these mechanisms to limit JET-ILW pedestal evolution.

The JET-ILW Pedestal- JET pulse 82585 (described in Ref. [4]) is part of an experimental campaign studying the effect of deuterium fueling on pedestal structure. In this series of discharges, the pedestal broadens but does not heighten with increasing fueling rate. This behavior is contrary to the expected $[7,8]$ scaling of pedestal width with plasma pressure - typically $\Delta \propto \sqrt{\beta_{p o l}}$, where $\Delta$ is the pedestal width, $\beta_{\text {pol }}=2 \mu_{0} P / B_{\text {pol }}^{2}, P$ is pressure and $B_{\text {pol }}$ is the poloidal magnetic field. In order to model the transport in this JET-ILW pedestal, we have reproduced the pre-ELM temperature and density profiles, along with the shape of the outer flux surface from data reported in Ref. [4]. The necessary gyrokinetic inputs (radial profiles of density and temperature, magnetic equilibrium, and background flow profile) are self-consistently constructed using the equilibrium code VMEC in conjunction with neoclassical formulas [20] for the bootstrap current and radial electric field. Figure 1 shows the density, temperature, and pressure profiles (a), along with $\eta_{e}=L_{n} / L_{T e}$ (b).

Pedestal instabilities - A host of instabilities has been identified in $\mathrm{H}$-mode pedestals both experimentally and in gyrokinetic simulations [21]. Experimentally, fluctuations have been diagnosed that are consistent with MTM [22, 23, 25], KBM [22, 24-26], and trapped electron modes (TEM) [22], while linear gyrokinetic modeling has identified MTM [19, 27-30], TEM [30, 31], ETG [29, 30, 32, 33], KBM [19, 26, 27, 29, 30, 34, 35], and unidentified drift waves [36]. Due to the large number of possible instabilities, and the complexity of the nonlinear turbulent state (that reflects the underlying instabilities in not so obvious ways), no clear picture has emerged regarding the relative importance of these modes for pedestal transport. The EPED model appeals 

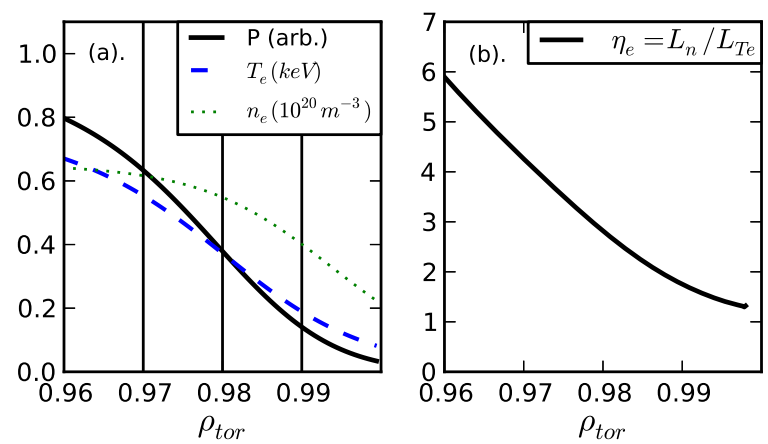

FIG. 1: Profiles of temperature, density, and pressure (a), and $\eta_{e}(\mathrm{~b})$.

exclusively to KBM as the salient local pedestal-limiting instability [7]. The identification of such a wide array of linear instabilities in gyrokinetic studies underscores the necessity of nonlinear simulations to determine with certainty the relevant transport mechanisms. Such an analysis will be given below following the present accounting of linear instabilities in the JET-ILW pedestal.

The observed modes may be classified into three distinct categories-MTM, KBM, and electrostatic drift waves (ESDW) (encompassing, e.g., ITG and TEM) with the following defining characteristics: 1) the KBM is identified by a positive (ion direction) frequency, ballooning parity (predominantly symmetric [antisymmetric] mode structure for $\phi\left[A_{\|}\right]$along the field line), and a near-vanishing $E_{\|}=-\partial_{z} \phi+i \omega A_{\|}$(expected for a mode largely determined by ideal MHD). The latter is quantified by the ratio

$$
\hat{E}_{\|}=\frac{\int d z\left|-\partial_{z} \phi+i \omega A_{\|}\right|}{\int d z\left|\partial_{z} \phi\right|+\int d z\left|i \omega A_{\|}\right|}
$$

where $\phi$ is the electrostatic potential, $z$ is the coordinate parallel to the background magnetic field, $\omega$ is the complex mode frequency, and $A_{\|}$is the parallel magnetic vector potential. KBM were determined to have values of $\hat{E}_{\|}$typically below $\left.0.1,2\right)$ The MTM is identified by mode structures with predominantly tearing parity (opposite of ballooning parity) and negative (electron direction) frequencies; the resulting transport is dominantly electromagnetic as quantified by the ratio $\left(Q_{e}^{E M} / Q^{E S}\right)$ where $Q_{e}^{E M}\left(Q^{E S}\right)$ is the electron electromagnetic (electrostatic) heat flux, all calculated from the linear eigenmodes, 3) all modes not encompassed by the MTM/KBM criteria are categorized as ESDW.

The Gene code was used in both its local and (more comprehensive) global modes of operation. Simulations included the Landau-Boltzmann collision operator, electromagnetic effects, and fully-realistic geometry. Parallel magnetic fluctuations are included in local but not global simulations. In global simulations, the effects of parallel magnetic fluctuations are approximated by setting the $\nabla B$ drift equal to the curvature drift - a treatment that has previously been demonstrated to accurately capture KBM stability and was verified to do so in this case with the local code. In the following, $z$ denotes the coordinate along the field line, $y$ denotes the binormal direction, and $x$ is the radial direction. The typical numerical resolution used for these linear simulations was $(48-96,48,16)$ grid points in the $\left(z, v_{\|}, \mu\right)$ coordinates, where $v_{\|}$is the parallel velocity and $\mu$ is the magnetic moment (proportional to perpendicular velocity squared). Typically 256 radial grid points (for a $\sim 46$ ion gyro radii box) were used for global simulations and $13 k_{x}$ wave numbers for local simulations.

Local linear simulations identify MTM as the dominant low- $k_{y}$ instability over most of the pedestal, with KBM unstable in the region $\rho_{\text {tor }}>0.988$. Notably, the MTM is only unstable at finite $k_{x}$ (analogous to the ballooning angle $\theta_{0}$ in ballooning theory) as seen in Fig. 2 (b). As a result, this MTM instability would be missed by analyses (often standard) sampling only $k_{x}=0$. Several tests were performed in order to conclusively identify the mode as MTM. As expected for MTM, the mode frequency is in the electron direction and very close to $\omega_{*}=-k_{y} \rho_{s} c_{s}\left(1 / L_{n}+1 / L_{T e}\right)$. Parameter scans determine that MTM growth rates increase strongly with electron temperature gradients, are weakly suppressed by density and ion temperature gradients, and are largely independent of collisionality $[28,37,38]$. The MTM exhibits moderate cancellation in $E_{\|}\left(\hat{E}_{\|} \gtrsim 0.3\right.$ being typical), although the electrostatic component of the mode is not crucial to its existence; the MTM growth rate smoothly decreases to $\sim 55 \%$ of its nominal value when the amplitude of the electrostatic potential is artificially reduced towards $\phi=0$. Other mode properties (e.g., $A_{\|}$ mode structure, and frequency) remain unchanged. However, when $A_{\|}$is analogously reduced, the MTM transforms to a qualitatively distinct ESDW with a growth rate reduced to $\sim 30 \%$ of the MTM growth rate.

A more-comprehensive global analysis also identifies the MTM as the dominant instability. In global simulations encompassing the entire pedestal the KBM is not manifest, while the MTM persists with growth rates roughly comparable to the radially-averaged local MTM growth rates. MTM growth rates decrease in the presence of $E \times B$ shear flow, but to a lesser degree than the surrounding ESDW as shown in Fig. 2 (a). The mode structure of the MTM eigenmode (shown in Fig. 4) elucidates the origin of its insensitivity to shear flow. The MTM magnetic vector potential $A_{\|}$peaks at the top and bottom of the tokamak where flux surfaces are broadly spaced, is radially localized, and has fine radial structure. All these features contribute to a lower effective shear rate [39], which will become important in the discussion of MTM-driven turbulence below.

Pedestal $\beta$ scan- In light of experimental uncertain- 

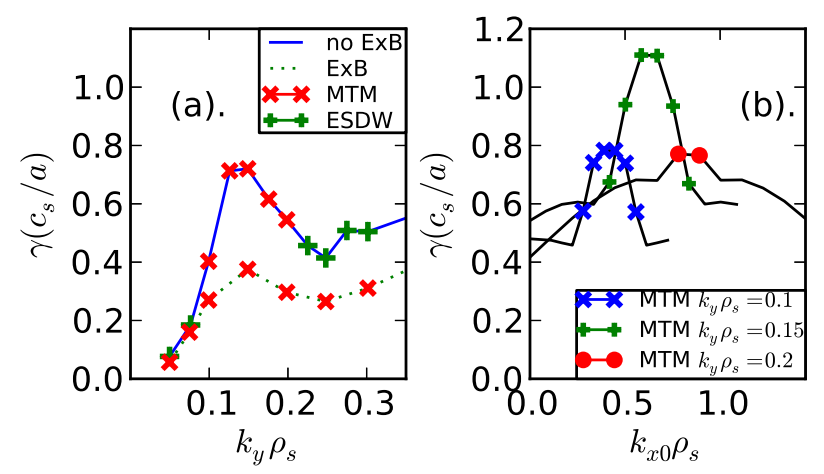

FIG. 2: Global growth rates with and without $E \times B$ shear (a), dependence of local MTM growth rates on ballooning angle (b).
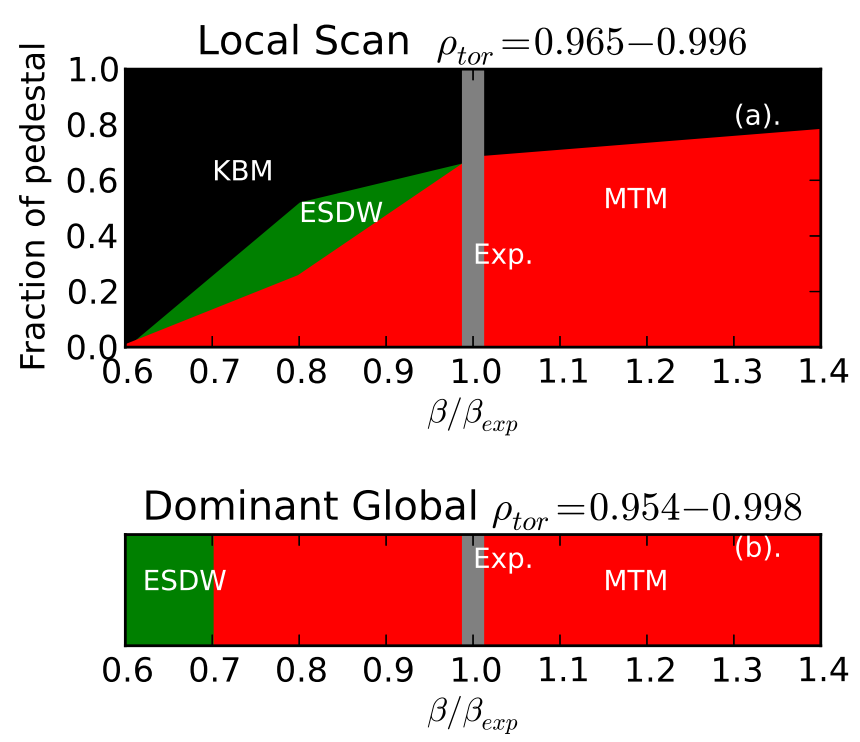

FIG. 3: Fraction of the pedestal unstable to KBM, MTM, and ESDW in local gyrokinetic simulations (a), and the dominant low- $k_{y}\left(k_{y} \rho_{s}<0.5\right)$ instability in global simulations (b) over a range of $\beta$ surrounding the experimental operating point. MTM becomes increasingly dominant as $\beta$ increases. MTM persists in global simulations, while $\mathrm{KBM}$ is subdominant. The scan uses fully self-consistent equilibria for each $\beta$ point.

ties and the dynamic nature of pedestal evolution, we repeat the above analysis while reconstructing fully selfconsistent equilibria for various values of $\beta$ about the nominal experimental value. In addition to gauging the sensitivity of our results, such a scan can be roughly conceptualized as sampling the inter-ELM evolution of the pedestal, during which the pressure profile recovers from an ELM collapse and returns to its pre-ELM level.

The $\beta$ scan was constructed by varying the pedestal top temperature from $60 \%$ to $140 \%$ of its nominal experimental value and producing fully self-consistent equilib- ria for each scan point. Local linear results, summarized in Fig. 3 (a), show the fraction of the pedestal over which $\mathrm{KBM}$ and MTM are unstable. KBM is identified across the entire pedestal in the low- $\beta$ regime but becomes more stable and limited to progressively narrower regions as $\beta$ increases towards and beyond the experimental value. In contrast, MTM becomes unstable at $\beta$ values slightly below the experimental operating point and becomes more pronounced as $\beta$ increases. This trend suggests that the MTM becomes more robustly unstable over the course of an ELM cycle, as would be expected for the dominant mode limiting profile evolution. In contrast, the KBM becomes increasingly stable and limited to a narrow region at the far edge. This picture of local KBM stability is consistent with other studies demonstrating that KBM is in the so-called second stability regime in JET [19] and NSTX [29] pedestal scenarios.

Global simulations further emphasize the role of MTM. In Fig. 3 (b), displaying the dominant global mode over the $\beta$ scan, MTM is seen to be dominant in a broad region of parameter space; at low $\beta$, it is replaced not by KBM but rather ESDW. In other words, a fully global treatment identifies KBM to be subdominant even in cases where a local analysis finds robust KBM instability across the entire pedestal. KBM instability can only be recovered in global simulations by increasing the simulation box (far beyond the width of the pedestal) while artificially maintaining steep gradients across the box. This suggests that the local analysis that is often relied upon to identify KBM stability boundaries may be inadequate. The suppression of a given mode in a global eigenvalue problem (as described above) even when it is found to be present (and unstable) in a local analysis, is not unique to KBM. Such a behavior is illustrated, for example, in Ref. [40] that deals with the global versus local stability for the magneto rotational instability. The complex reasons why it happens for some modes and not others will be dealt with in a more detailed publication.

It should be noted that the gyrokinetic model does not include the kink term. Although it can be ruled out as a potential effect, the term was not important when implemented in a gyrokinetic framework for the pedestal scenario examined in Ref. [34, 35].

Nonlinear turbulent transport- Having characterized the linear instabilities at and near the experimental operating point, one must calculate their consequences for the nonlinear turbulent state. Of particular importance is the question whether nonlinear turbulent transport produced by the MTM can account for experimental power balance, and by extension the crucial properties of the pedestal. To this end, extensive nonlinear simulations were performed. GENE was used primarily in a local mode of operation, employing a real space radial coordinate with Dirichlet boundary conditions, while keeping plasma parameters and gradients constant across the box. This approach was benchmarked against global sim- 


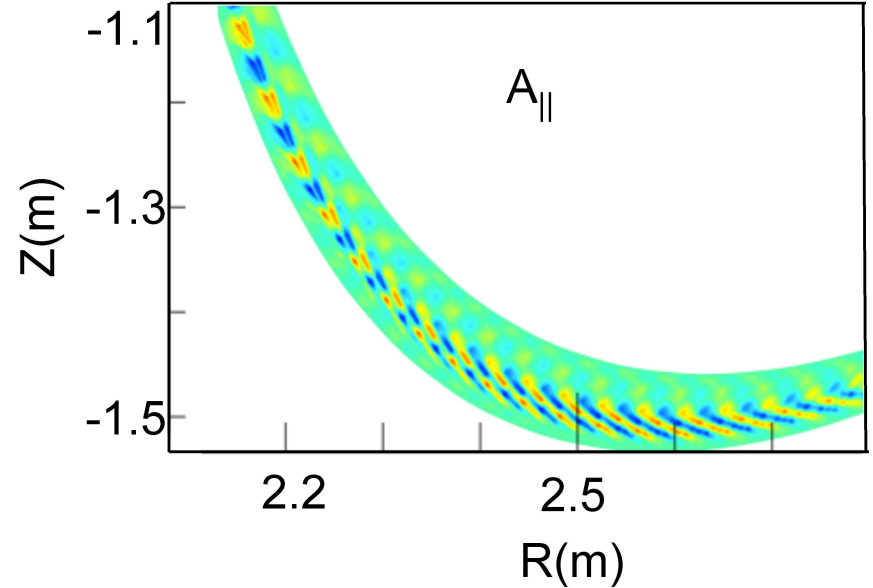

FIG. 4: Structure of $A_{\|}$for a linear global MTM eigenmode without shear flow. The eigenmode peaks at the top and bottom (shown) of the tokamak, is limited to a narrow radial band, and is characterized by fine-scale radial strucutre, serving to reduce the effect of flow shear on the MTM.

ulations where possible; a nonlinear global simulation with $k_{y, \min }=0.05$ produced transport levels differing by only $\sim 10 \%$ from the corresponding local simulation, consistent with earlier work suggesting MTM is not strongly affected by global effects [41]. However, nonlinear global simulations could not be extended to lower $k_{y}$ due to numerical issues (likely resolvable with higher resolution simulations that are beyond the scope of this work). Simulations used $(256,48,48,16)$ gridpoints in the $\left(x, z, v_{\|}, \mu\right)$ coordinates, values established by extensive convergence tests. Transport quantities were most sensitive to resolution in the $y$ coordinate, converging at $k_{y, \min } \rho_{s}=0.018$, while resolving up to to $k_{y, \max }=1.55$.

As expected for MTM-driven turbulence, nonlinear simulations show that heat flux is predominantly in the electron electromagnetic channel. All comparisons indicate that the turbulence is the nonlinear manifestation of the underlying MTM instability. The insensitivity of the MTM to shear suppression is also observed in the nonlinear simulations; The turbulence was evolved shear-free to a saturated state at which point the shear was turned on. The shear flow reduced the electrostatic component (ESDW) of the flux by orders of magnitude while the electromagnetic component (MTM) was reduced by a factor of $\sim 2$, roughly consistent with the decrease in growth rates shown in Fig. 2 (a).

Three radial locations (denoted with vertical lines in Fig. 1 (a)) were selected for detailed analysis: $\rho_{\text {tor }}=0.97$ near the pedestal top, $\rho_{\text {tor }}=0.98$ in the steep gradient region, and $\rho_{t o r}=0.99$ at the far edge. The far outer point produced transport levels approximately three times lower than nominal power balance. Higher transport could certainly be achieved with small modifications to the background profiles, but such a course was

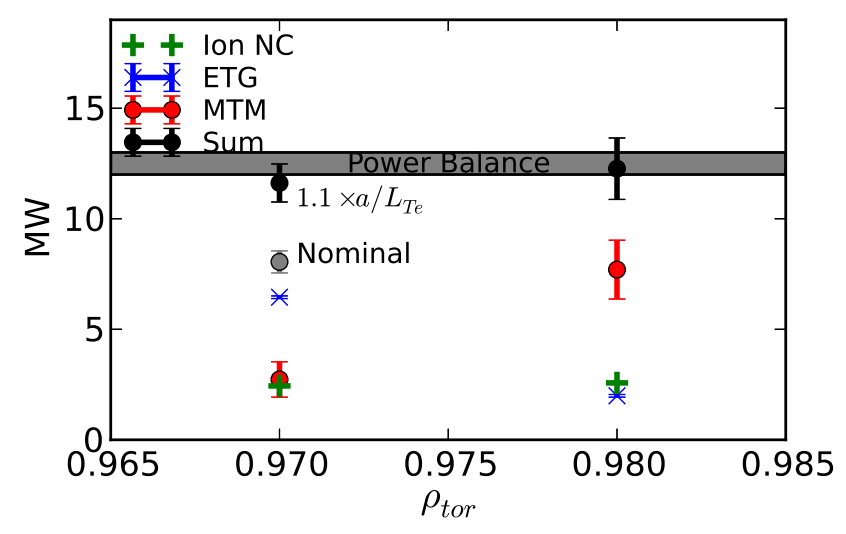

FIG. 5: Contributions to the heat transport from ion neoclassical (green pluses), ETG (blue Xs), and MTM (red circles) at two radial locations, demonstrating agreement with experimental power balance.

not pursued due to the severe uncertainties intrinsic to this far edge region (e.g., potential power losses due to charge exchange, uncertainty in separatrix position, and effects associated with close proximity to the open field line region). Simulation results at the other radial locations reproduce experimental power balance strikingly well as shown in Fig. 5. MTM-dominated turbulence at $\rho_{\text {tor }}=0.98$ produces $\sim 7.5 \mathrm{MW}$ of transport, single-scale simulations of ETG turbulence produce an additional $\sim 2$ MW, and calculations of neoclassical transport predict $2.5 \mathrm{MW}$ (ions). The combination amounts to $\sim 12 \mathrm{MW}$, compared to $12-13 \mathrm{MW}$ estimated experimentally (16 MW neutral beam heating and 3-4 MW radiation losses in the core). Simulations at $\left(\rho_{\text {tor }}=0.97\right)$ are dominated by ETG transport (due to the large value of $\eta_{e}$ ) with MTM and neoclassical playing a smaller role. Power balance is again satisfied, requiring only a minor adjustment (10\% increase) to the electron temperature gradient.

Summary and Discussion- We demonstrate that the combination of MTM and ETG turbulence is sufficient to account for power balance across most of the JET-ILW pedestal. Electrostatic ion-scale instabilities are strongly shear suppressed, while KBM is insignificant in a broad region of parameter space surrounding the experimental operating point. This work is perhaps the first study to demonstrate agreement between transport predictions of nonlinear gyrokinetic simulations and experimental pedestal transport levels, establishing the capacity of gyrokinetics to model critical aspects of pedestal transport. Additionally, this work lays a foundation for continued study of the JET-ILW pedestal, notably, the trends related to fueling levels and impurity content that impede JET-ILW discharges from recovering high confinement levels.

Acknowledgements.- This research used resources of 
the National Energy Research Scientific Computing Center, a DOE Office of Science User Facility; the HELIOS supercomputer system at the International Fusion Energy Research Centre, Aomori, Japan; and the Texas Advanced Computing Center (TACC) at The University of Texas at Austin. This work was supported by U.S. DOE Contract No. DE-FG02-04ER54742. This work has been carried out within the framework of the EUROfusion Consortium and has received funding from the Euratom research and training programme 2014-2018 under grant agreement No 633053. The views and opinions expressed herein do not necessarily reflect those of the European Commission.

[1] F. Wagner et al., Phys. Rev. Lett. 491408 (1982).

[2] E. J. Doyle et al., Nucl. Fusion 47, S18 (2007).

[3] J. W. Connor, Plasma Phys. Control. Fusion 40, 531 (1998).

[4] M. J. Leyland, M. N. A. Beurskens, L. Frassinetti, C. Giroud, S. Saarelma, P. B. Snyder, J. Flanagan, S. Jachmich, M. Kempenaars, P. Lomas, G. Maddison, R. Neu, I. Nunes, and K. J. Gibson, Nucl. Fusion, 55, 013019 (2015).

[5] C. F. Maggi, S. Saarelma, F. J. Casson, C. Challis, E. de la Luna, L. Frassinetti, C. Giroud, E. Joffrin, J. Simpson, M. Beurskens, I. Chapman, J. Hobirk, M. Leyland, P. Lomas, C. Lowry, I. Nunes, F. Rimini, A.C.C. Sips, and H. Urano, Nucl. Fusion, 55, 113031 (2015).

[6] C. Giroud et al., Plasma Phys. Control. Fusion, 57, 035004 (2015).

[7] P. B. Snyder, N. Aiba, M. Beurskens, R. J. Groebner, L. D. Horton, A. E. Hubbard, J. W. Hughes, G. T. A. Huysmans, Y. Kamada, and A. Kirk, Nucl. Fusion, 49, 085035, (2009).

[8] P. B. Snyder, R. J. Groebner, A. W. Leonard, T. H. Osborne, and H. R. Wilson, Phys. Plasmas 16, 056118 (2009).

[9] F. Jenko, W. Dorland, M. Kotschenreuther, and B. N. Rogers, Phys. Plasmas, 7, 1904 (2000).

[10] T. Görler, X. Lapillonne, S. Brunner, T. Dannert, F. Jenko, F. Merz, and D. Told, Journal of Computational Physics 230, 7053 (2011).

[11] R. D. Hazeltine et al., Phys. Fluids 18, 1778 (1975).

[12] D. J. Applegate et al., Phys. Plasmas 11, 5085 (2004).

[13] H. Doerk, F. Jenko, M. J. Pueschel, and D. R. Hatch, Phys. Rev. Lett., 106, 155003 (2011).

[14] W. Guttenfelder, J. Candy, S. M. Kaye, W. M. Nevins, E. Wang, R. E. Bell, G. W. Hammett, B. P. LeBlanc, D. R. Mikkelsen, and H. Yuh, Phys. Rev. Lett., 106, 155004 (2011).

[15] W. Horton, B. G. Hong, and W. M. Tang, Phys. Fluids 31,2971 (1988).

[16] W. Dorland, F. Jenko, M. Kotschenreuther, and B. N. Rogers, Phys. Rev. Lett. 85, 5570 (2000).

[17] F. Jenko and W. Dorland, Phys. Rev. Lett. 89, 225001 (2002).

[18] W. M. Nevins et al., Phys. Plasmas, 13, 122306 (2006).

[19] S. Saarelma, M. N. A. Beurskens, D. Dickinson, L. Frassinetti, M. J. Leyland, C. M. Roach, and E.-J. Con- tributors, Nucl. Fusion, 53, 123012 (2013).

[20] O. Sauter, C. Angioni, and Y. R. Lin-Liu, Physics of Plasmas, 6, 28342839 (1999).

[21] R. J. Groebner et al., Nucl. Fusion, 53, 093024 (2013).

[22] D. R. Smith, R. J. Fonck, G. R. McKee, D. S. Thompson, R. E. Bell, A. Diallo, W. Guttenfelder, S. M. Kaye, B. P. LeBlanc, and M. Podesta, Phys. Plasmas, 20, 055903 (2013).

[23] P. Manz, J. E. Boom, E. Wolfrum, G. Birkenmeier, I. G. J. Classen, N. C. L. Jr, U. Stroth, and the ASDEX Upgrade Team, Plasma Phys. Control. Fusion, 56, 035010 (2014).

[24] Z. Yan, G. R. McKee, R. J. Groebner, P. B. Snyder, T. H. Osborne, and K. H. Burrell, Phys. Rev. Lett., 107, 055004 (2011).

[25] Z. Yan, G. R. McKee, R. J. Groebner, P. B. Snyder, T. H. Osborne, M. N. Beurskens, and K. H. Burrell, Phys. Plasmas, 18, 056117 (2011).

[26] A. Diallo, J. W. Hughes, M. Greenwald, B. LaBombard, E. Davis, S.-G. Baek, C. Theiler, P. Snyder, J. Canik, J. Walk, T. Golfinopoulos, J. Terry, M. Churchill, A. Hubbard, M. Porkolab, L. Delgado-Aparicio, M. L. Reinke, A. White, and Alcator C-Mod team, Phys. Rev. Lett., 112, 115001 (2014).

[27] D. Dickinson, C. M. Roach, S. Saarelma, R. Scannell, A. Kirk, and H. R. Wilson, Phys. Rev. Lett., 108, 135002 (2012).

[28] D. Dickinson, C. M. Roach, S. Saarelma, R. Scannell, A. Kirk, and H. R. Wilson, Plasma Phys. Control. Fusion, 55, 074006 (2013).

[29] J. M. Canik, W. Guttenfelder, R. Maingi, T. H. Osborne, S. Kubota, Y. Ren, R. E. Bell, H. W. Kugel, B. P. LeBlanc, and V. A. Souhkanovskii, Nucl. Fusion, 53, 113016 (2014).

[30] D. R. Hatch, D. Told, F. Jenko, H. Doerk, M. G. Dunne, E. Wolfrum, E. Viezzer, T. A. U. Team, and M. J. Pueschel, Nucl. Fusion, 55, 063028 (2015).

[31] D. P. Fulton, Z. Lin, I. Holod, and Y. Xiao, Phys. Plasmas 21, 042110 (2014).

[32] D. Told, F. Jenko, P. Xanthopoulos, L. D. Horton, E. Wolfrum, and ASDEX Upgrade Team, Phys. Plasmas 15102306 (2008).

[33] F. Jenko, D. Told, P. Xanthopoulos, F. Merz, and L.D. Horton, Phys. Plasmas 16055901 (2009).

[34] W. Wan, S. E. Parker, Y. Chen, Z. Yan, R. J. Groebner, and P. B. Snyder, Phys. Rev. Lett., 109, 185004, (2012).

[35] W. Wan, S. E. Parker, Y. Chen, R. J. Groebner, Z. Yan, A. Y. Pankin, and S. E. Kruger, Phys. Plasmas, 20, 055902 (2013).

[36] E. Wang, X. Xu, J. Candy, R. J. Groebner, P. B. Snyder, Y. Chen, S. E. Parker, W. Wan, G. Lu, and J. Q. Dong, Nucl. Fusion, 52, 103015 (2012).

[37] I. Predebon and F. Sattin, Phys. Plasmas 20040701 (2013).

[38] D. Carmody, M. J. Pueschel, and P. W. Terry, Phys. Plasmas 20052110 (2013).

[39] T. S. Hahm and K. H. Burrell, Phys. Plasmas, 2, 1648 (1995).

[40] S. M. Mahajan and V. Krishan, The Astrophysical Journal, 682, 602 (2008).

[41] H. Doerk, F. Jenko, T. Grler, D. Told, M. J. Pueschel, and D. R. Hatch, Phys. Plasmas, 19, 055907 (2012). 